\title{
Morbilidad oftalmológica en escolares de comunidades rurales en Venezuela
}

\section{Ophthalmologic morbidity in schoolchildren of rural communities in Venezuela}

\author{
Dr. Victor Agüin, ${ }^{\mathrm{I}}$ Lic. Ligia Elena Cisneros, ${ }^{\mathrm{I}}$ Lic. Rosa Melendez ${ }^{\text {II }}$ \\ ${ }^{\text {I }}$ Hospital Universitario de Caracas. Venezuela \\ II Universidad de Carabobo. Venezuela
}

\section{RESUMEN}

Introducción: el deterioro de la función visual limita el desarrollo adecuado de los niños y repercute en el rendimiento escolar.

Objetivo: determinar la frecuencia de morbilidad oftálmica en escolares que asisten a las escuelas ubicadas en las comunidades rurales de Puerto Cabello, Venezuela.

Métodos: estudio descriptivo de tipo transversal en 1183 escolares mediante un cuestionario elaborado para tal fin.

Resultados: se registraron 1183 diagnósticos, de los cuales $981(82,94 \%)$ no mostraron ninguna enfermedad oftálmica, y los restantes $202(17,06 \%)$ se evaluaron como patológicos. Las ametropías constituyeron la enfermedad más frecuentemente encontrada, $10,65 \%$, seguido por conjuntivitis en el 4,05\% de los casos.

Conclusiones: Hay una alta incidencia de enfermedades oftalmológicas que repercuten en el desarrollo integral del niño y su adaptación al medio escolar. Se recomienda la implementación de programas de detección o pesquisa de enfermedades oftalmológicas en escolares y profundizar en las acciones de educación y orientación a las familias y a la población en general.

Palabras clave: Oftalmología, escolares, morbilidad, comunidades rurales, ametropía. 


\section{ABSTRACT}

Introduction: the deterioration of visual function limits the appropriate development of children and affects school performance.

Objective: to determine the prevalence of ophthalmic morbidity in students attending schools in rural communities of Puerto Cabello, Venezuela.

Methods: a descriptive cross-sectional study performed on 1183 schoolchildren through a customized questionnaire for this purpose.

Result: there were 1183 diagnoses, of which 981 (82.94\%) did not show any ophthalmologic disease, and the remaining $202(17.06 \%)$ were evaluated as pathological. Ametropia was the most frequent pathology found in $10.65 \%$, followed by conjunctivitis in $4.05 \%$ of cases.

Conclusions: high incidence of ophthalmologic diseases that affect the comprehensive development of children and their adaptation to the school environment was found. Finally, it was recommended to implement programs of detection or screening of ophthalmologic diseases in schoolchildren, and to delve into the educational and orientation actions aimed at families and general population

Key words: Ophthalmology, schoolchildren, morbidity, rural communities, ametropia.

\section{INTRODUCCIÓN}

De los cinco sentidos que los humanos poseen, es la visión la que está más relacionada con la comunicación y las relaciones sociales. ${ }^{1}$ La ausencia de la función visual origina consecuencias adversas en los estudiantes, limita su desarrollo y repercute en su rendimiento escolar. ${ }^{2}$ La detección temprana de las alteraciones oftálmicas permiten promover la salud visual, prevenir enfermedades oculares y reducir la ceguera prevenible o curable. ${ }^{1}$

El periodo crítico en el desarrollo de la visión se extiende desde el nacimiento hasta los 10 años. La etapa más precoz de la infancia es una etapa maleable, es decir que tanto se puede ganar como perder función visual. Pero progresivamente se va llegando a una etapa en la que es más difícil ganar visión, hasta convertirse en la rigidez del adulto, que ya es irreversible. De aquí la importancia de la edad para realizar un diagnostico precoz y un tratamiento oportuno. ${ }^{3}$

Los problemas visuales no diagnosticados son una de las principales causas de fracaso escolar. ${ }^{1,3,4} \mathrm{~A}$ pesar de esta situación, no existen programas de prevención o control para las enfermedades oculares; los existentes son pocos y han sido desarrollados en medios urbanos. ${ }^{3}$ De ahí la importancia de la detección de las alteraciones visuales y afecciones oculares en los niños escolares, quienes por estar en pleno desarrollo de sus facultades físicas e intelectuales, se convierten en una población de especial interés para el estudio de los defectos de la visión. ${ }^{5}$

En este sentido, se desea determinar la frecuencia de morbilidad oftálmica en escolares que asisten a las escuelas ubicadas en las comunidades rurales de Puerto Cabello, Venezuela, con el objetivo principal de prevenir, corregir problemas físicos, funcionales y de orden psicológico que afectarían grandemente su rendimiento académico y adaptación social ulterior. 


\section{MÉTODOS}

Se realizó un estudio descriptivo de corte transversal. El grupo en estudio estuvo integrada por escolares de 6 a 12 años de edad pertenecientes a las comunidades rurales de Puerto Cabello, Venezuela, en el periodo comprendido entre septiembre a noviembre de 2010 .

El tamaño de la muestra se calculó a partir de un universo de 3871 alumnos, para un nivel de confianza de $95 \%$ (error $\alpha=0,05$ ). Se realizo un muestreo estratificado polietápico, que se inició con la selección aleatoria de las poblaciones rurales ubicada en Puerto Cabello, Venezuela. Posteriormente se eligieron las escuelas primarias ubicadas en dicha población. El número de alumnos que debía seleccionarse en cada escuela se calculó como parte proporcional de la muestra a partir de la proporción que representaba esa escuela en la población de estudio.

Se incluyeron 1423 alumnos, a los cuales se les proporcionó una carta de consentimiento informado que explicaba la prueba a realizar y donde se garantizaba el anonimato y la confidencialidad de la información. Participaron en el estudio 1183 preescolares.

Se informó a las autoridades de las distintas escuelas sobre las actividades realizadas y se solicitó su autorización. Se proporcionaría a los representantes información sobre los resultados oftalmológicos en caso de ser identificada alguna enfermedad, para su posterior atención en el sector de la salud.

Como instrumento de recolección se utilizo un cuestionario sometido a procedimiento de pilotaje previo y se evaluó la confiabilidad de las respuestas.

El cuestionario presentaba dos secciones: la primera con datos de identificación y la segunda fue destinada para la exploración oftalmológica completa. La metodología para el examen ocular utilizada fue la siguiente:

- Examen de agudeza visual mediante la cartilla de Snellen, adecuada para una distancia de cinco metros; para el caso de escolares que aún no habían aprendido a leer, se procedió a utilizar la cartilla con los ganchos de Pluger.

- El examen clínico con retinoscopio, para determinar las anomalías de la refracción.

- Examen de fondo de ojo en los niños en los cuales se encontraban alteraciones de la visión no explicables por trastornos de la refracción o acomodación.

La recolección de datos se realizó por parte de los investigadores con la finalidad de reducir el error en la medición y evitar variabilidad entre observadores. Asimismo, se contó con la colaboración de un oftalmólogo, quien se encargó de la realización de los exámenes especializados a la población escolar en estudio.

Los datos fueron registrados en tablas elaboradas previamente para tal fin y procesados estadísticamente con la asistencia de programas computarizados.

http://scielo.sld.cu 


\section{RESULTADOS}

Durante el período de estudio se evaluaron 1183 escolares, de los cuales el 55,68\% fueron del género masculino y el $44,28 \%$ del femenino. El grupo de edad que predominó fue de 8 a 9 años con el 38,71\%, seguido del grupo de 6 a 7 años con $33,05 \%$. La distribución según edad y género se muestra en la tabla 1.

Tabla 1. Distribución según edad y género en escolares de comunidades rurales. Puerto Cabello, 2010

\begin{tabular}{|c|c|c|c|c|c|c|}
\hline \multirow{3}{*}{$\begin{array}{l}\text { Grupo de edad } \\
\text { (años) }\end{array}$} & \multicolumn{4}{|c|}{ Género } & & \\
\hline & \multicolumn{2}{|c|}{ Masculino } & \multicolumn{2}{|c|}{ Femenino } & \multicolumn{2}{|c|}{ Total } \\
\hline & $\mathrm{n}$ & $\%$ & $n$ & $\%$ & $\mathrm{n}$ & $\%$ \\
\hline $6-7$ & 203 & 18,42 & 188 & 14,62 & 391 & 33,05 \\
\hline $8-9$ & 234 & 21,25 & 224 & 17,45 & 458 & 38,71 \\
\hline $10-12$ & 176 & 16,01 & 158 & 12,21 & 334 & 28,23 \\
\hline Total & 614 & 55,68 & 569 & 44,28 & 1183 & 100,0 \\
\hline
\end{tabular}

Se registraron 1183 diagnósticos, de los cuales 981 (82,94\%) no mostraron ninguna enfermedad oftálmica, y los restantes 202 (17,06 \%) diagnósticos se evaluaron como patológicos. Las ametropías constituyen la afección más frecuentemente encontrada en $10,65 \%$, seguido por conjuntivitis con el $4,05 \%$ de los casos. Se detectaron otras enfermedades como: blefaritis $(1,01 \%)$, estrabismo $(0,67 \%)$, pingueculitis $(0,50 \%)$, celulitis preseptal $(0,50 \%)$ ptosis congénita $(0,16 \%)$ y leucoma con $0,08 \%$ (tabla 2 ).

Tabla 2. Distribución porcentual de la morbilidad oftalmológica según diagnostico realizado y la edad del escolar de comunidades rurales. Puerto Cabello 2010

\begin{tabular}{|l|c|c|c|c|}
\hline \multirow{2}{*}{ Diagnóstico } & \multicolumn{2}{|c|}{ Grupo de edad (años) } & \multirow{2}{*}{ Total } \\
& $6-7$ & $8-9$ & $10-12$ & \\
\hline Sano & 82,92 & 82,93 & 82,96 & 82,94 \\
\hline Ametropías & 10,69 & 10,64 & 10,62 & 10,65 \\
\hline Conjuntivitis & 4,08 & 4,07 & 4,00 & 4,05 \\
\hline Blefaritis & 1,01 & 0,80 & 1,03 & 1,01 \\
\hline Estrabismo & 0,65 & 0,66 & 0,66 & 0,67 \\
\hline Pingueculitis & 0,53 & 0,53 & 0,44 & 0,50 \\
\hline Celulitis preseptal & 0,53 & 0,44 & 0,53 & 0,50 \\
\hline Ptosis congénita & 0,48 & 0,0 & 0,0 & 0,16 \\
\hline \hline Leucoma & 0,0 & 0,24 & 0,0 & 0,08 \\
\hline
\end{tabular}

http://scielo.sld.cu 


\section{DISCUSIÓN}

Las enfermedades oculares originan una minusvalía grave en escolares, que afecta directamente la capacidad de aprendizaje, incidiendo negativamente en el desarrollo integral del niño y por consiguiente en el bajo rendimiento escolar. ${ }^{6}$

Si se considera que el proceso de aprendizaje se basa en un sistema que incluye como primera etapa el proceso de ingreso o percepción donde juegan un rol determinante los sistemas sensoriales, principalmente visión y audición, ${ }^{4}$ la identificación precoz de las alteraciones en estos sentidos debe ser un objetivo prioritario de la pediatría, oftalmología preventiva y de la atención primaria en salud, de tal manera que favorezca una buena o aceptable integración del niño en sus tareas escolares. ${ }^{7}$

En la investigación se encontró que el 17,06 \% de los escolares estudiados tenían algún tipo de alteración ocular, cifra que se considera elevada en relación a otros estudios. ${ }^{8-10}$ Lo anterior podría deberse al descuido de los padres de familia respecto a la salud de sus hijos, precaria situación económica que obliga a esperar una solución natural de la enfermedad, falta de información de los padres acerca de las enfermedades oculares o una conjunción de todas estas causas. ${ }^{6}$

Las estimaciones presentadas permiten establecer una idea acerca de la magnitud de estos problemas. Si los datos obtenidos, son extrapolados a otras zonas del país, en especial a las que poseen características similares a la estudiada, se debe pensar que la magnitud de los problemas oculares en nuestro país es alta y desconocida en su mayor proporción.

Pese a este panorama (cuya percepción no escapa a las autoridades de salud), las afecciones oftálmicas ha sido poco estudiada en nuestro medio. Existen reportes que corroboran que en las comunidades rurales de Latinoamérica la magnitud de estos problemas es elevada. ${ }^{11,12}$

Los vicios de refracción (ametropías) constituyen la enfermedad más frecuente en el estudio aportando $10,65 \%$ de los casos, tanto en forma general como en los diferentes grupos de edad, en completa concordancia con la literatura. $7,8,10$ Gonzáles Del Aguila, en una casuística de escolares de zonas rurales con afecciones oftalmológicas del departamento del Cuzco, Perú, refiere que los problemas de refracción representan el $12,8 \%$ de las alteraciones detectadas; valores cercano al hallado en este estudio. ${ }^{13}$

Los vicios de refracción se presentan como una causa importante de limitación en la edad escolar, si se toma en consideración el proceso enseñanza aprendizaje. ${ }^{4,6,10}$ En este sentido, es importante recordar que los pediatras juegan un papel importante en la detección de este tipo de alteración fácilmente prevenible y curable. El personal médico debe enseñar a los padres que el niño debe tener una valoración oftalmológica en diferentes momentos de su desarrollo: 1. Período neonatal. 2. A los tres años. 3. A los cinco años y cada tres años a partir de esa edad. ${ }^{2}$

Asimismo, es necesario que los programas de Atención Primaria en Salud de nuestro país, incluyan un acápite dedicado a la valoración oftalmológica básica, que haga énfasis en la detección de problemas de agudeza visual. De esta manera, los médicos que están en contacto cotidiano con el grueso de la población, pueden realizar rutinariamente esta evaluación y se conviertan en los descubridores 
iniciales de estas alteraciones y puedan proceder inmediatamente a su referencia a los centros de salud adecuados para su tratamiento y manejo especializado.

Las afecciones conjuntivales ocupan el 4,05 \% de las enfermedades, es la segunda en frecuencia, lo que demuestra la existencia de una acción del medio ambiente como factor predisponente o desencadenante de estas entidades. Sin embargo, no se debe olvidar que las conjuntivitis se pueden presentar solas o como manifestaciones de una afección sistémica. ${ }^{14}$

Afortunadamente existe poca frecuencia de estrabismo en la investigación (0,50 \%), en contraste con algunas publicaciones, ${ }^{10,12}$ situación que nos satisface por la gran dificultad del tratamiento y la relatividad del pronostico cuando no es detectado a tiempo.

Finalmente, se recomienda que los resultados del presente estudio, estimulen la investigación de estos problemas e impulsen a las autoridades y organizaciones de salud a implementar programas para profundizar las acciones de educación y orientación a los padres de familia y a la población en general.

\section{REFERENCIAS BIBLIOGRÁFICAS}

1. Tenaglia R. Ambliopia, su pesquisa en la escuela. Archivo Argentino Pediatria. $2002 ; 100(4): 342-4$.

2. Kohler L, Stigmar G. Testing for hypermetropia in the school vision screening programme. Acta Ophthalmol. 1981;59:369.

3. Diaz R, Raimann RM, Farina A. Pesquisa en la escuela. Archivo Argentino Pediatria. 2002;100(4):342-4.

4. Ondategui Parra JC, Borras Garcia MR, Castane Farran M, Pacheco Cutillas. Manual de exámenes clínicos. Mexico: Alfaomega; 2001.

5. Garrido M, Manrique G. Atención Primaria en Oftalmologia. Trujillo: Universidad Nacional de la Libertad; 2001.

6. Bueno M. Detección precoz de los defectos sensoriales y del desarrollo. An Esp Pediatr. 2006;27:31-2.

7. Loman J, Quinn GE, kamoun L. Ying GS, Maguire MG, Hudesman D, et al. Darkness and near work: myopia and its progression in third-year law students. Ophthalmology. 2002;09(5):1032-8. Comment in: Ophthalmology. 2004;110(6):1069-70; author reply 1071-2.

8. Zharo J, Xianjun P. Refractive error study in children: results from shunyi District, China. Am J Ophthalmol. 2006;129(4):427-35.

9. Castiella Acha JC, Pastor Jimeno JC. Ametropias. Su correcion. En: Castiella Archa IC, Pastor Jimeno JC, editores. La refracción en el niño. Madrid: Mcgraw Hill interamericana; 2004. p. 97-104.

http://scielo.sld.cu 
10. Delgado M, Nguyen N, Cox T, Singh K, Lee D. Automated perimetry: a report by the American Academy of Ophthalmology. Ophthalmology. 2007;109:2362-74.

11. Badia JA, De Vechi HP, Alezzandrini AA, Gaisiner PD, Gonella A, Gordillo CH, et al. Fundamentos de oftalmología. Buenos Aires: El Ateneo; 2003.

12. De Amorim CC, Orefice F, Dutra G, Souza D, Ramalho M. Disminución de la agudeza visual y ametropias en la población estudiantil universitaria. An Soc Mex Oftalmol 2003;50(1):29-41.

13. Gonzáles del Águila C. Crónica y Reflexiones sobre la Oftalmología en el Peru. Rev Per Oftalm. 2001;14(1):

14. Riemann C, Hanson Foster J. A comparison of manual kinetic and automated static perimetry in obtaining ptosis fields. Arch Ophthalmol. $2004 ; 118 ; 65-9$.

Recibido: 30 de abril de 2011.

Aprobado: 28 de noviembre de 2011.

Victor Agüin. Urb San Jose de Tarbes. Res. Tarbes "B". Apart 9-A, Valencia. Carobobo, Venezuela.

Correo electrónico: vinote@hotmail.com 